\title{
NUMERICAL SIMULATION OF FLOW AND HEAT TRANSFER IN HYDROMAGNETIC MICROPOLAR FLUID BETWEEN TWO STRETCHABLE DISKS WITH VISCOUS DISSIPATION EFFECTS
}

\author{
Kashif Ali, Shahzad Ahmad, Muhammad Ashraf \\ Centre for Advanced Studies in Pure and Applied Mathematics, Bahauddin Zakariya University, Multan, Pakistan \\ e-mail: muhammadashraf@bzu.edu.pk
}

A study of magnetohydrodynamic (MHD) flow with viscous dissipation and heat transfer in an electrically conducting laminar steady viscous incompressible micropolar fluid between two infinite uniformly stretching disks is presented. The transformed self similar nonlinear ODEs are first linearized using a quasi linearization method and then solved by employing a combination of a direct and an iterative method. The study may be beneficial to flow and thermal control of polymeric processing.

Keywords: MHD, micropolar fluid, stretchable disk, viscous dissipation, couple stress, heat transfer rate

\section{Introduction}

The exploration in the field of flow over a stretching surface has attracted attention of the research community due to its significant applications in different industries such as extrusion paper production, extrusion of polymers sheet, metal and plastic industries (Altan et al., 1979; Fisher, 1976; Tadmor and Klein, 1970). The problem of fluid flow between parallel disks is also important due to its applications in many technological and engineering processes. These applications include semiconductor-manufacturing processes with rotating wafers, magnetic storage devices, gas turbine engines, hydrodynamical machines and apparatus, crystal growth processes, rotating machinery, biomechanics, geothermal, geophysical, heat and mass exchanges, computer storage devices, viscometry, lubrication, oceanography radial diffusers, etc. Robert et al. (2010) presented the analytical solution of axi-symmetric flow between two infinite stretching disks whereas Fang and Zhang (2008) found the exact solution for the axi-symmetric flow between two stretchable infinite disks. Munawar et al. (2011) studied flow of an incompressible viscous fluid between two continuously stretching coaxial disks by employing the optimal HAM. Xinhui et al. (2012) studied asymmetric flow and heat transfer of a viscous fluid between contracting/expanding rotating disks by using the homotopy analysis method.

All the above cited researchers are, however, confined to the flow and heat transfer problems of classical Newtonian fluids. The Newtonian model is, however, inadequate to completely describe some modern scientific, engineering and industrial processes which involve materials possessing an internal structure. The scope of non-Newtonian fluids has significantly increased mainly due to their connection with applied sciences. The governing equations of motion for non-Newtonian fluids are highly nonlinear and complicated as compared to those for Newtonian fluids. The flow problems of non-Newtonian fluids are challenging for researchers due to their inherent complexity. Hoyt and Fabula (1964) predicted experimentally that fluids having polymeric additives display a significant reduction of shear stress and polymeric concentration (see Eringen, 1965). Deformation of such materials can be well explained by the theory of micropolar fluids given by Eringen $(1964,1966)$. Micropolar fluids have applications in colloidal fluids flow, 
blood flows, dumbbell molecules or short rigid cylindrical elements, liquid crystals, lubricants, turbulent shear flow and flow in capillaries, fluid suspensions, animal blood, fluid with bar like elements, heat and mass exchangers, etc. The steady laminar incompressible flow of a micropolar fluid between two parallel disks in which the lower disk is taken to be impermeable while the upper one is permeable was discussed numerically by Ashraf et al. (2009a). The magnetohydrodynamics (MHD) has attracted the research community due to its novel industrial applications. Rashidi et al. (2014) investigated velocity and temperature profiles as well as entropy generation in magnetohydrodynamic (MHD) and slip flow over a rotating porous disk with different properties using numerical methods. Neetu (2014) found the analytical solution to magnetohydrodynamic flow problem of an incompressible micropolar fluid between two eccentrically disks. MHD steady and axisymmetric flow of an incompressible viscous fluid between two radially stretching sheets was analyzed by Hayat and Nawaz (2010). Hayat et al. (2011) examined a time dependent magnetohydrodynamic (MHD) flow problem of a micropolar fluid between two radially stretching infinite sheets.

The above cited researchers did not take the effects of viscous dissipation in their investigations. Therefore, the aim of the present study is to investigate MHD steady viscous incompressible electrically conducting micropolar fluid flow and heat transfer between two stretching disks in the presence of a transverse magnetic field and viscous dissipation effects.

\section{Problem formulation}

Consider hydromagnetic steady laminar viscous flow and heat transfer of an incompressible electrically conducting micropolar fluid between two stretchable infinite disks located at $z=-L$ and $z=L$ as shown in Fig. 1. A uniform transverse magnetic field $\underline{B}$ is applied perpendicularly at the disks. The geometry of the problem suggests that the cylindrical polar coordinate system is most suitable for the study. Both the disks are stretched uniformly with the velocity proportional to the $r$ coordinate. The magnetic Reynolds number is assumed to be small, and hence the induced magnetic field can be neglected as compared to the imposed magnetic field (Shercliff, 1965). We assume that there is no applied polarization voltage, so the electric field is zero. The components of velocity $(u, v, w)$ and microrotation $\left(v_{1}, v_{2}, v_{3}\right)$ along the radial, transverse and axial directions can be written respectively as

$$
\begin{array}{lll}
u_{r}=u_{r}(r, z) & u_{\theta}=0 & u_{z}=u_{z}(r, z) \\
v_{1}=0 & v_{2}=v_{2}(r, z) & v_{3}=0
\end{array}
$$

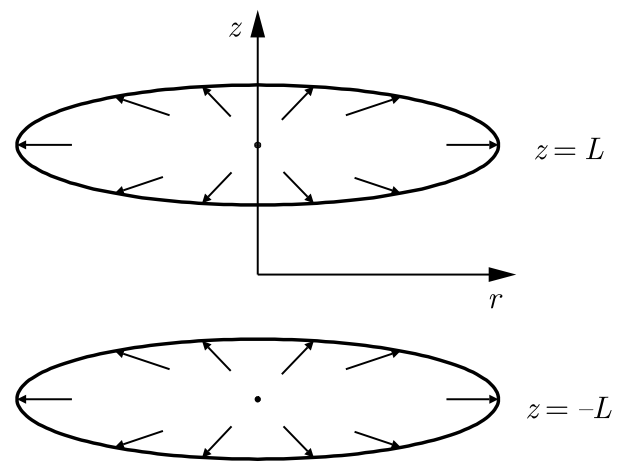

Fig. 1. Physical configuration

Following the work of Eringen $(1964,1966)$ and in view of Eq. $(2.1)$, the governing equations of the problem under consideration can be written as 


$$
\frac{u_{r}}{r}+\frac{\partial u_{r}}{\partial r}+\frac{1}{L} \frac{\partial u_{z}}{\partial \eta}=0
$$

and

$$
\begin{aligned}
& \rho\left(u_{r} \frac{\partial u_{r}}{\partial r}+\frac{u_{z}}{L} \frac{\partial u_{r}}{\partial \eta}\right)=-\frac{\partial p}{\partial r}-\frac{\kappa}{L} \frac{\partial v_{2}}{\partial \eta}+(\mu+\kappa)\left(\frac{\partial^{2} u_{r}}{\partial r^{2}}+\frac{1}{r} \frac{\partial u_{r}}{\partial r}-\frac{u_{r}}{r^{2}}+\frac{1}{L^{2}} \frac{\partial^{2} u_{r}}{\partial \eta^{2}}\right)-\sigma_{e} B_{0}^{2} u_{r} \\
& \rho\left(u_{r} \frac{\partial u_{z}}{\partial r}+\frac{u_{z}}{L} \frac{\partial u_{z}}{\partial \eta}\right)=-\frac{1}{L} \frac{\partial p}{\partial \eta}+\kappa\left(\frac{\partial v_{2}}{\partial r}+\frac{v_{2}}{r}\right)+(\mu+\kappa)\left(\frac{\partial^{2} u_{z}}{\partial r^{2}}+\frac{1}{r} \frac{\partial u_{z}}{\partial r}+\frac{1}{L^{2}} \frac{\partial^{2} u_{z}}{\partial \eta^{2}}\right) \\
& \rho j\left(u_{r} \frac{\partial v_{2}}{\partial r}+\frac{u_{z}}{L} \frac{\partial v_{2}}{\partial \eta}\right)=\kappa\left(\frac{1}{L} \frac{\partial u_{r}}{\partial \eta}-\frac{\partial u_{z}}{\partial r}\right)-2 \kappa v_{2}+\gamma\left(\frac{\partial^{2} v_{2}}{\partial r^{2}}-\frac{v_{2}}{r^{2}}+\frac{1}{r} \frac{\partial v_{2}}{\partial r}+\frac{1}{L^{2}} \frac{\partial^{2} v_{2}}{\partial \eta^{2}}\right)
\end{aligned}
$$

where $\eta=z / L$ is the similarity variable, $\rho$ is density, $p$ is pressure, $\mu$ is dynamic viscosity of the fluid, $\kappa$ is vortex viscosity, $j$ is microinertia, $\gamma$ is spin gradient viscosity, $\sigma_{e}$ is electrical conductivity, $B_{0}$ is strength of the magnetic field. Including viscous dissipation effects, the energy equation for the problem of flow between two stretching disks can be written as

$$
\rho c_{p}\left(u_{r} \frac{\partial T}{\partial r}+\frac{u_{z}}{L} \frac{\partial T}{\partial \eta}\right)-k_{0}\left(\frac{1}{L^{2}} \frac{\partial^{2} T}{\partial \eta^{2}}+\frac{\partial^{2} T}{\partial r^{2}}+\frac{1}{r} \frac{\partial T}{\partial r}\right)-\frac{\mu}{L^{2}}\left(\frac{\partial u_{r}}{\partial \eta}\right)^{2}=0
$$

where $T$ is temperature, $c_{p}$ is specific heat capacity and $k_{0}$ is thermal conductivity of the fluid.

The boundary conditions for the problem may be written as,

$$
\begin{array}{llll}
u_{r}(r,-L)=r E & u_{r}(r, L)=r E & u_{z}(r,-L)=0 & u_{z}(r, L)=0 \\
\nu_{2}(r,-L)=0 & \nu_{2}(r, L)=0 & T(r,-L)=T_{1} &
\end{array}
$$

where $E$ is the parameter determining stretching strength of both the upper and lower disks, having units of $1 / t$.

Partial differential Eqs. (2.3) and (2.4) can be converted into ordinary ones by using the following similarity transformations

$$
u_{r}=-\frac{r E}{2} f^{\prime}(\eta) \quad u_{z}=E L f(\eta) \quad v_{2}=-\frac{E r}{2 L^{2}} g(\eta) \quad \theta(\eta)=\frac{T-T_{2}}{T_{1}-T_{2}}
$$

where $T_{1}$ and $T_{2}$ are temperatures at the lower and upper disks, respectively. We see that the velocity field given in Eq. (2.6) identically satisfies continuity Eq. (2.1), and hence represents possible fluid motion. By using Eq. (2.6) in Eqs. (2.3) and (2.4), we get the following nonlinear ordinary differential equations in dimensionless form

$$
\begin{aligned}
& \left(1+C_{1}\right) f^{\prime \prime \prime \prime}-C_{1} g^{\prime \prime}-\operatorname{Re} f f^{\prime \prime \prime}-\operatorname{Re} M^{2} f^{\prime \prime}=0 \\
& C_{3} g^{\prime \prime}+C_{1}\left(f^{\prime \prime}-2 g\right)+\operatorname{Re} C_{2}\left(\frac{f^{\prime} g}{2}-f g^{\prime}\right)=0 \\
& \theta^{\prime \prime}+\frac{1}{4} \operatorname{PrEc} f^{\prime \prime 2}-\operatorname{Re} \operatorname{Pr} f \theta^{\prime}=0
\end{aligned}
$$

where $\operatorname{Re}=\left(\rho E L^{2}\right) / \mu$ is the stretching Reynolds number, $M=\sqrt{\left(\sigma_{e} B_{0}^{2}\right) /(\rho E)}$ is the magnetic parameter, $C_{1}=\kappa / \mu$ is the vortex viscosity parameter, $C_{2}=j / L^{2}$ is the microinertia density parameter, $C_{3}=\gamma / \mu L^{2}$ is the spin gradient viscosity parameter, $\operatorname{Pr}=\left(\mu c_{p}\right) / k_{0}$ is the Prandtl number and $\mathrm{Ec}=\left(r^{2} E^{2}\right) /\left[c_{p}\left(T_{1}-T_{2}\right)\right]$ is the Eckert number.

Boundary conditions given in Eq. $(2.7)_{2}$ also get the form

$$
\begin{aligned}
& f(-1)=f(1)=0 \quad f^{\prime}(-1)=-2 \quad f^{\prime}(1)=-2 \\
& g(-1)=0 \quad g(1)=0 \quad \theta(-1)=1 \quad \theta(1)=0
\end{aligned}
$$




\section{Computational procedure}

In this paper, we discuss the approach based on quasi-linearization of nonlinear ODEs.

\subsection{Quasi-linearization}

We use quasi-linearization to construct sequences of vectors $\left\{f^{(k)}\right\},\left\{g^{(k)}\right\}$, and $\left\{\theta^{(k)}\right\}$, which converge to the numerical solutions to Eqs. (2.7), respectively. To construct $\left\{f^{(k)}\right\}$, we linearize Eq. $(2.7)_{1}$ by retaining only the first order terms as follows:

We set

$$
G\left(f, f^{\prime}, f^{\prime \prime}, f^{\prime \prime \prime}, f^{\prime \prime \prime \prime}\right) \equiv\left(1+C_{1}\right) f^{\prime \prime \prime \prime}-C_{1} g^{\prime \prime}-\operatorname{Re} f f^{\prime \prime \prime}-\operatorname{Re} M^{2} f^{\prime \prime}
$$

and

$$
\begin{aligned}
& G\left(f^{(k)}, f^{\prime(k)}, f^{\prime \prime(k)}, f^{\prime \prime \prime}(k), f^{\prime \prime \prime \prime}(k)\right)+\left(f^{(k+1)}-f^{(k)}\right) \frac{\partial G}{\partial f^{(k)}}+\left(f^{\prime(k+1)}-f^{\prime(k)}\right) \frac{\partial G}{\partial f^{\prime(k)}} \\
& \quad+\left(f^{\prime \prime(k+1)}-f^{\prime \prime(k)}\right) \frac{\partial G}{\partial f^{\prime \prime(k)}}+\left(f^{\prime \prime \prime(k+1)}-f^{\prime \prime \prime(k)}\right) \frac{\partial G}{\partial f^{\prime \prime \prime}(k)}+\left(f^{\prime \prime \prime \prime \prime(k+1)}-f^{\prime \prime \prime \prime}(k)\right) \frac{\partial G}{\partial f^{\prime \prime \prime \prime}(k)}=0
\end{aligned}
$$

which simplifies to

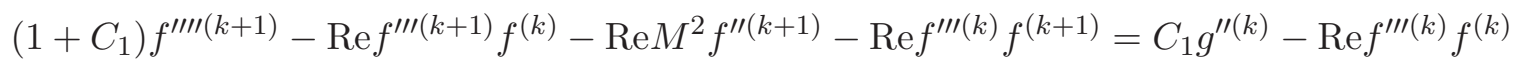

Now Eq. (3.1) gives a system of linear differential equations with $f^{k}$ being the numerical solution vector of the $k^{\text {th }}$ equation. To solve the linear ODEs, we replace the derivatives with their central difference approximations, giving rise to the sequence $\left\{f^{(k)}\right\}$ generated by the following linear system

$$
B f^{(k+1)}=C \quad \text { with } \quad B \equiv B_{n \times n}\left(f^{(k)}\right) \quad \text { and } \quad C \equiv C_{n \times 1}\left(f^{(k)}\right)
$$

where $n$ is the number of grid points. On the other hand, Eqs. $(2.7)_{2,3}$ are linear in $g$ and $\theta$ respectively and, therefore, in order to generate the sequences $\left\{g^{(k)}\right\}$ and $\left\{\theta^{(k)}\right\}$, we write

$$
\begin{aligned}
& C_{3} g^{\prime \prime(k+1)}+C_{1}\left(f^{\prime \prime(k+1)}-2 g^{(k+1)}\right)+\operatorname{Re} C_{2}\left(\frac{f^{\prime(k+1)} g^{(k+1)}}{2}-g^{\prime(k+1)} f^{(k+1)}\right)=0 \\
& \theta^{\prime \prime(k+1)}+\frac{1}{4} \operatorname{PrEc} f^{\prime \prime(k+1)^{2}}-\operatorname{RePr} f^{(k+1)} \theta^{\prime(k+1)}=0
\end{aligned}
$$

Importantly, $f^{(k+1)}$ is considered to be known in the above equation and its derivatives are approximated by the central differences.

We outline the computational procedure as follows:

- Provide the initial guess $f^{(0)}, g^{(0)}$ and $\theta^{(0)}$, satisfying the boundary conditions given in Eq. (2.8)

- Solve the linear system given by Eq. (3.2) to find $f^{(1)}$

- Use $f^{(1)}$ to solve the linear system arising from the FD discritization of Eqs. (3.3), to get $g^{(1)}$ and $\theta^{(1)}$

- Take $f^{(1)}, g^{(1)}$ and $\theta^{(1)}$ as the new initial guesses and repeat the procedure to generate the sequences $\left\{f^{(k)}\right\},\left\{g^{(k)}\right\}$ and $\left\{\theta^{(k)}\right\}$ which, respectively, converge to $f, g$ and $\theta$ (the numerical solutions to Eqs. (2.7) 
- The three sequences are generated until

$$
\max \left\{\left\|f^{(k+1)}-f^{(k)}\right\|_{L_{\infty}},\left\|g^{(k+1)}-g^{(k)}\right\|_{L_{\infty}},\left\|\theta^{(k+1)}-\theta^{(k)}\right\|_{L_{\infty}}\right\}<10^{-6}
$$

It is important to note that the coefficient matrix $B$ in Eq. (3.2) will be pentadiagonal and not diagonally dominant, and hence the iterative method (like SOR) may fail or work very poorly. Therefore, some direct method like LU factorization or Gaussian elimination with full pivoting (to ensure stability) may be employed. On the other hand, Eqs. (3.3) will give a rise to the diagonally dominant algebraic system when discretized using the central differences, which allows us to use the SOR method. Lastly, we may also improve the order of accuracy of the solution by using the polynomial extrapolation scheme.

\section{Results and discussion}

In this Section, the results are presented in tabular and graphical forms together with their discussion and interpretations. Our objective is to develop a better understanding of the effects of the micropolar structure of fluids on flow and heat transfer characteristics. The parameters of the study are the Reynolds number Re, the magnetic parameter $M$, the micropolar parameters $C_{1}, C_{2}$, and $C_{3}$, the Eckert number Ec and the Prandtl number Pr. The physical quantities of our interest are the shear stress, the couple stress and the heat transfer rate at the disks which are, respectively, proportional to $f^{\prime}(-1), g^{\prime}(-1), \theta^{\prime}(-1)$ and $\theta^{\prime}(1)$. It is important to note that $f^{\prime \prime}(-1)=f^{\prime \prime}(1), g^{\prime}(-1)=g^{\prime}(1)$ and $\theta^{\prime}(-1)=\theta^{\prime}(1)$ for Ec $=0$ due to symmetry of the problem. But in the case when Ec $\neq 0$, the symmetry of temperature profiles no longer exists, and thus $\theta^{\prime}(-1) \neq \theta^{\prime}(1)$ as $C_{3}$ affects the temperature distribution only (clear from decoupled Eqs. (2.7). Therefore, in the presence of viscous dissipation, we will consider $f^{\prime \prime}(-1), g^{\prime}(-1), \theta^{\prime}(-1)$ and $\theta^{\prime}(1)$ as well. We shall study the effects of the parameters described above on $f^{\prime \prime}(-1), g^{\prime}(-1), \theta^{\prime}(-1)$ and $\theta^{\prime}(1)$ as well as on the velocity profiles $f(\eta), f^{\prime}(\eta)$, the microrotation profile $g(\eta)$ and the temperature profile $\theta(\eta)$.

The sets of values of the dimensionless micropolar parameters $C_{1}, C_{2}$ and $C_{3}$ used in the present work are given in Table 1 . In order to establish the validity of our numerical computations and to improve the order of accuracy of the solutions, numerical values of radial velocity $f^{\prime}(\eta)$ are computed for three grid sizes $h, h / 2$ and $h / 4$ and then Richardson extrapolation is used as presented in Table 2. It also shows the convergence of our numerical results as the step size decreases. Table 3 shows that the shear and couple stresses increase, where the heat transfer rate increases at the upper disk and decreases at the lower disk as the stretching Reynolds number increases. The increased stretching rate of the disks forces the fluid to move rapidly towards the disks, thus increasing both the shear and couple stresses. Moreover, the fluid is carrying away the heat from the flow region, resulting in an increase in the temperature difference and, hence, the heat transfer rate.

Table 1. Five cases of values of micropolar parameters $C_{1}, C_{2}$ and $C_{3}$

\begin{tabular}{|c|c|c|c|}
\hline Case No. & $C_{1}$ & $C_{2}$ & $C_{3}$ \\
\hline \hline 1(Newtonian) & 0 & 0 & 0 \\
\hline 2 & 0.5 & 0.1 & 0.2 \\
\hline 3 & 1 & 0.3 & 0.4 \\
\hline 4 & 3 & 0.5 & 0.6 \\
\hline 5 & 5 & 0.7 & 0.8 \\
\hline
\end{tabular}


Table 2. Dimensionless radial velocity $f^{\prime}(\eta)$ on three grid sizes and extrapolated values for $\operatorname{Re}=15, M=1.5, C_{1}=3, C_{2}=0.5, C_{3}=0.6, \operatorname{Pr}=0.7, \mathrm{Ec}=0.5$

\begin{tabular}{|c|c|c|c|c|}
\hline \multicolumn{5}{|c|}{$f^{\prime}(\eta)$} \\
\hline$\eta$ & $\begin{array}{c}1^{\text {st }} \text { grid } \\
(h=0.02)\end{array}$ & $\begin{array}{c}2^{\text {nd }} \text { grid } \\
(h=0.01)\end{array}$ & $\begin{array}{c}3^{\text {rd }} \text { grid } \\
(h=0.005)\end{array}$ & $\begin{array}{c}\text { Extrapolated } \\
\text { values }\end{array}$ \\
\hline \hline-1 & -1.995047 & -1.998750 & -1.999686 & -1.999998 \\
\hline-0.6 & 0.092943 & 0.093467 & 0.093599 & 0.093642 \\
\hline-0.2 & 0.606570 & 0.607070 & 0.607195 & 0.607237 \\
\hline 0 & 0.652236 & 0.652728 & 0.652851 & 0.652892 \\
\hline 0.2 & 0.606570 & 0.607070 & 0.607195 & 0.607237 \\
\hline 0.6 & 0.092943 & 0.093467 & 0.093599 & 0.093642 \\
\hline 1 & -1.995047 & -1.998750 & -1.999686 & -1.999998 \\
\hline
\end{tabular}

Table 3. The effect of the stretching Reynolds number on the shear and couple stresses as well as the heat transfer rate with $M=1.5, C_{1}=3, C_{2}=0.5, C_{3}=0.6, \operatorname{Pr}=0.7, \mathrm{Ec}=0.5$

\begin{tabular}{|c|c|c|c|c|}
\hline $\mathrm{R}$ & $f^{\prime \prime}(-1)$ & $g^{\prime}(-1)$ & $\theta^{\prime}(-1)$ & $-\theta^{\prime}(1)$ \\
\hline \hline 0 & 5.245037 & 5.710461 & 0.556018 & 1.556018 \\
\hline 5 & 6.656467 & 6.023234 & 0.515410 & 2.005796 \\
\hline 10 & 7.898473 & 6.213057 & 0.453647 & 2.412162 \\
\hline 15 & 8.998815 & 6.329128 & 0.385683 & 2.777904 \\
\hline 20 & 9.986100 & 6.399389 & 0.317821 & 3.110079 \\
\hline
\end{tabular}

Table 4 shows that the magnetic parameter increases both the shear and couple stresses while reducing the heat transfer rate at the disks. From the mechanical point of view, the magnetic field exerts a friction like force, called the Lorentz force, which tends to drag the fluid

Table 4. The effect of the magnetic parameter on the shear and couple stresses as well as the heat transfer rate with $\operatorname{Re}=15, C_{1}=3, C_{2}=0.5, C_{3}=0.6, \operatorname{Pr}=0.7, \mathrm{Ec}=0.5$

\begin{tabular}{|c|c|c|c|c|}
\hline$M$ & $f^{\prime \prime}(-1)$ & $g^{\prime}(-1)$ & $\theta^{\prime}(-1)$ & $-\theta^{\prime}(1)$ \\
\hline \hline 0 & 6.270359 & 5.982139 & 0.798720 & 3.468070 \\
\hline 0.4 & 6.509053 & 6.014097 & 0.721225 & 3.365199 \\
\hline 0.8 & 7.172924 & 6.101533 & 0.558495 & 3.132747 \\
\hline 1.2 & 8.142989 & 6.225003 & 0.427947 & 2.903393 \\
\hline 1.6 & 9.300534 & 6.364672 & 0.382388 & 2.746286 \\
\hline
\end{tabular}

towards the disks. This not only results in increasing the shear stress at the disks but also causes greater spinning of the micro fluid particles, and hence increases the couple stress as well. Furthermore, the frictional force tends to raise the fluid temperature and thus decreases the temperature difference between the fluid and the disks. Therefore, the heat transfer rate, which is directly proportional to the temperature difference, also decreases. The influence of the micropolar parameters $C_{1}, C_{2}$ and $C_{3}$ on the shear and couple stresses is given in Table 5 . The first case corresponds to the Newtonian fluid whereas the remaining ones are taken arbitrarily to investigate their influence on the flow as chosen in the literature (Ashraf and Batool, 2013; Ali et al., 2014, 2009b). It may be concluded that the micropolar structure of the fluid tends to decrease the shear stress, which is in accordance with the experimental prediction of Hoyt and Fabula (1964) that the micro fluid particles cause significant reduction in the shear stress near a rigid body. Moreover, the particles also cause microrotation in the fluid, which is responsible for the couple stress at the disks, as shown in Table 5 . It is also clear from the table that the role 
Table 5. The effect of micropolar parameters on the shear and couple stresses as well as the heat transfer rate with $\operatorname{Re}=1, M=1.5, \operatorname{Pr}=2, \mathrm{Ec}=0.2$

\begin{tabular}{|c|c|c|c|c|}
\hline Cases & $f^{\prime \prime}(-1)$ & $g^{\prime}(-1)$ & $\theta^{\prime}(-1)$ & $-\theta^{\prime}(1)$ \\
\hline \hline 1 & 7.014810 & 0.000000 & 0.697019 & 1.960602 \\
\hline 2 & 6.473276 & 3.912051 & 0.698798 & 1.973223 \\
\hline 3 & 6.190115 & 3.896368 & 0.704141 & 1.984454 \\
\hline 4 & 5.478203 & 6.449680 & 0.729788 & 2.024384 \\
\hline 5 & 5.183083 & 7.439771 & 0.745661 & 2.046135 \\
\hline
\end{tabular}

of microfluid particles in increasing the heat transfer rate is not as pronounced as compared to its effect on the shear and couple stresses. Table 6 shows that the viscous dissipation may cause thermal reversal at the lower disk, thus decreasing the temperature of the fluid which, in turn, increases the temperature difference between the fluid and the upper disk, and hence the heat transfer rate at the upper disk.

Table 6. The effect of viscous dissipation on the heat transfer rate with $\operatorname{Re}=20, M=1.5$, $C_{1}=3, C_{2}=0.5, C_{3}=0.6, \operatorname{Pr}=0.3$

\begin{tabular}{|c|c|c|}
\hline Ec & $-\theta^{\prime}(-1)$ & $-\theta^{\prime}(1)$ \\
\hline \hline 0.0 & 0.844799 & 0.844799 \\
\hline 0.2 & 0.608888 & 1.080710 \\
\hline 0.4 & 0.372977 & 1.316622 \\
\hline 0.6 & 0.137066 & 1.552533 \\
\hline 0.8 & -0.098845 & 1.788444 \\
\hline
\end{tabular}

Now we present a graphical interpretation of our results. Streamlines for the present problem are given in Fig. 2. It is obvious that the streamlines near the walls are very close to each other showing larger gradients of the stream function which, in turn, predicts a greater fluid velocity

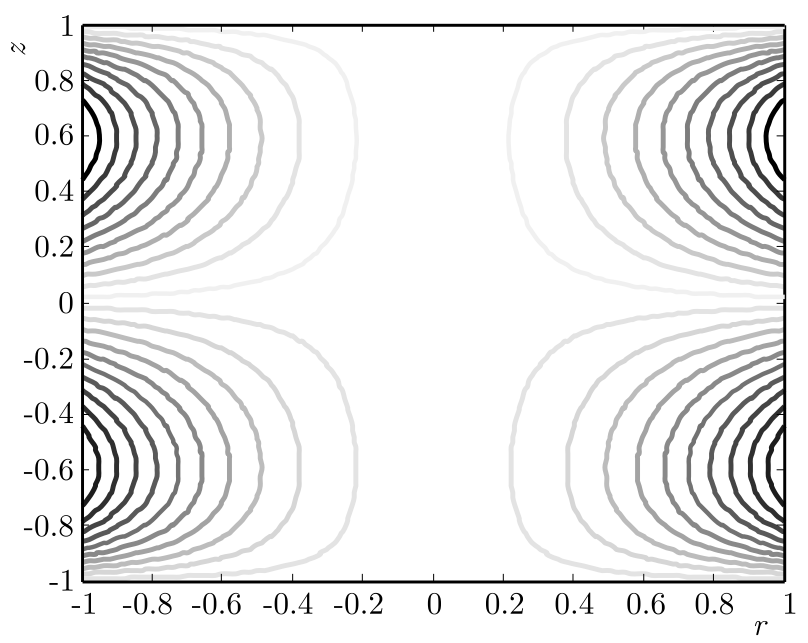

Fig. 2. Variation of streamlines for $\operatorname{Re}=5, M=1.5, C_{1}=3, C_{2}=0.5, C_{3}=0.6$

closer to the disks. In order to further validate the presented solution method, we consider the case when the distance between the disks is infinite and the upper disk is at rest. In this situation, the problem reduces to the micropolar fluid flow over a stretchable disk which was studied by Ashraf and Batool (2013). Figure 3 shows an excellent comparison of our numerical results with those of Ashraf and Batool (2013). 


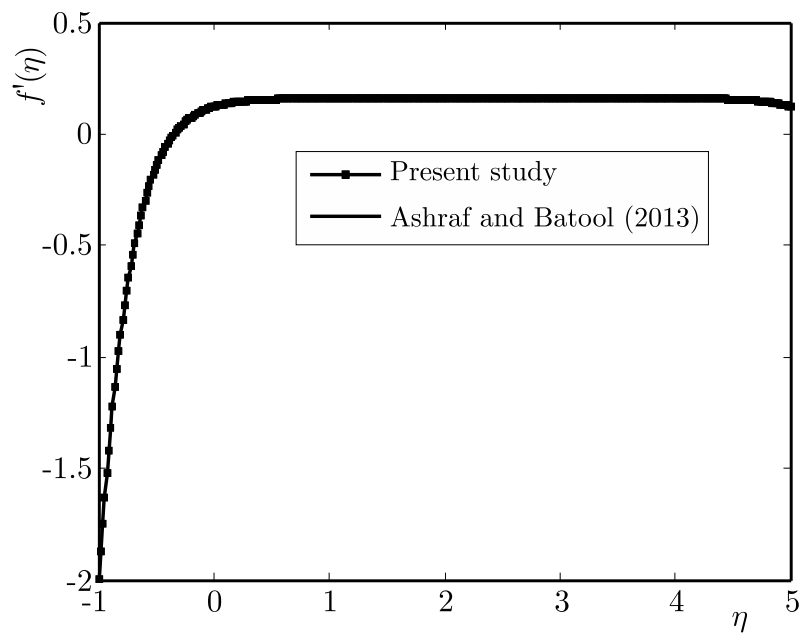

Fig. 3. Comparison with the results by Ashraf and Batool (2013)

Figures 4-6 show the influence of the magnetic parameter $M$ for typical values of the stretching Reynolds number, the micropolar parameters, the Eckert number and the Prandtl number. The magnetic parameter decreases the velocity as well as the microrotation distribution across the disks (Fig. 4 and 5). On the other hand, the external magnetic field decreases the thermal

(a)

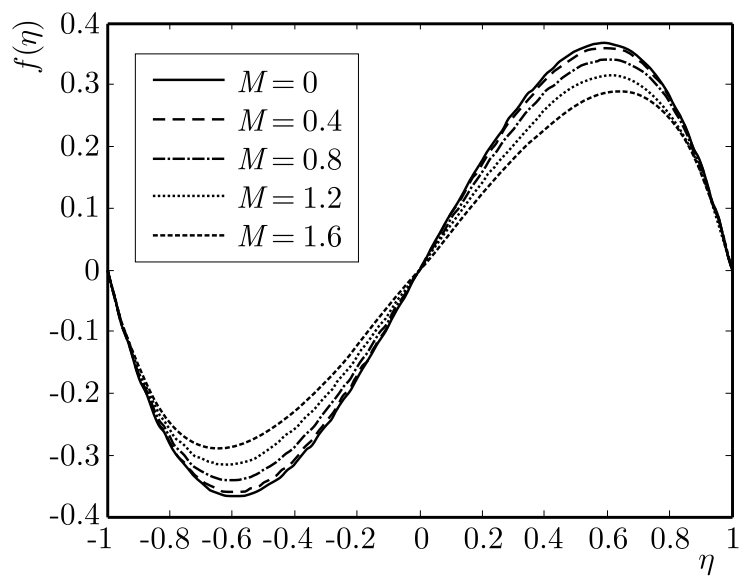

(b)

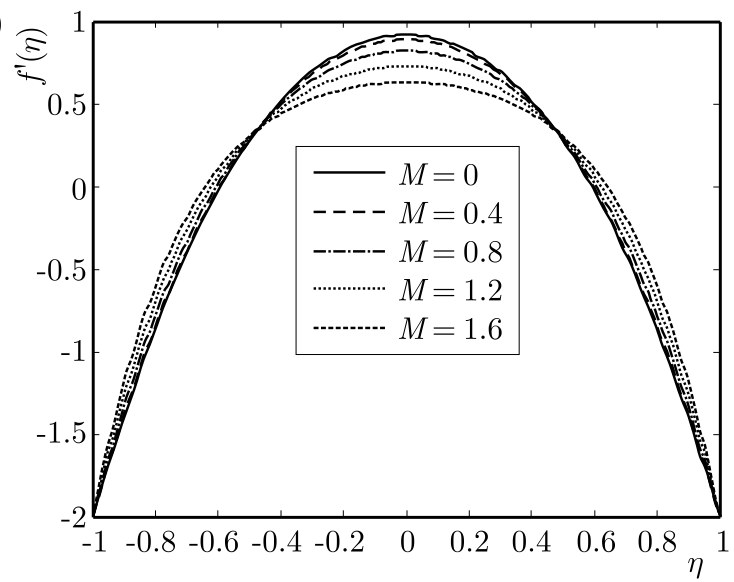

Fig. 4. Variation of (a) axial, (b) radial velocity for $\operatorname{Re}=15, C_{1}=3, C_{2}=0.5, C_{3}=0.6, \operatorname{Pr}=0.7$, $\mathrm{Ec}=0.5$ and various $M$

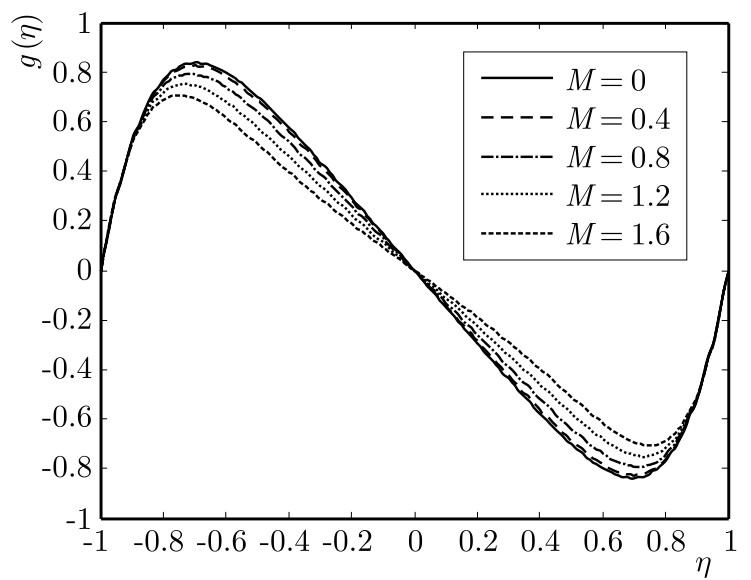

Fig. 5. Variation of microrotation for $\operatorname{Re}=15, C_{1}=3, C_{2}=0.5, C_{3}=0.6, \operatorname{Pr}=0.7, \mathrm{Ec}=0.5$ and various $M$ 
reversal by decreasing the temperature distribution across the disks, whether we consider the viscous dissipation effects or not, as shown in Fig. 6a.

(a)

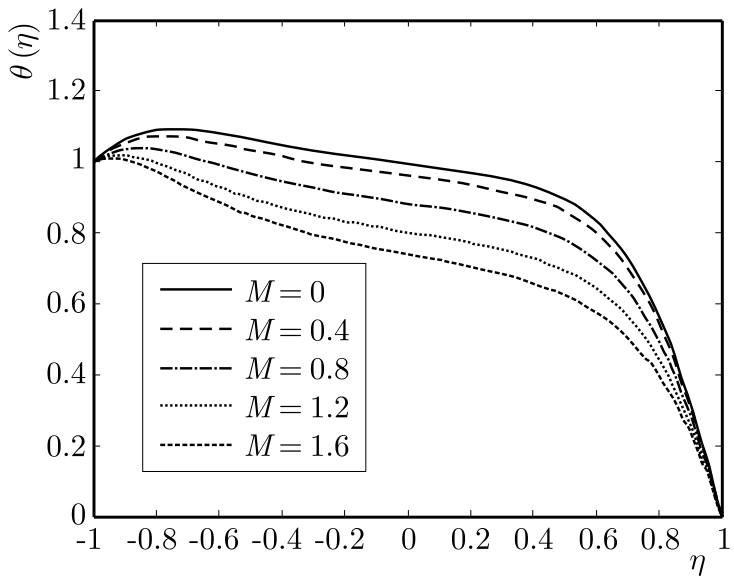

(b)

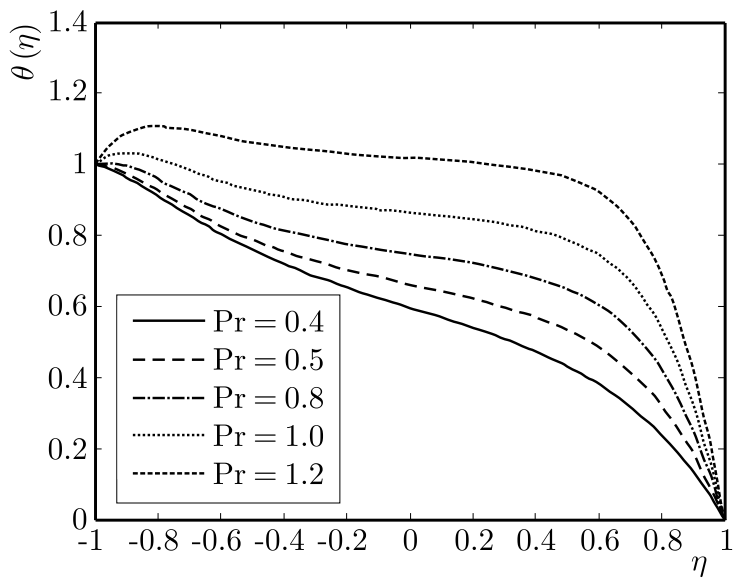

Fig. 6. Variation of temperature for (a) $\operatorname{Re}=15, C_{1}=3, C_{2}=0.5, C_{3}=0.6, \operatorname{Pr}=0.7, \mathrm{Ec}=0.5$ and various $M$, (b) $\mathrm{Re}=15, M=1.5, C_{1}=3, C_{2}=0.5, C_{3}=0.6, \mathrm{Ec}=0.4$ and various $\operatorname{Pr}$

We have noted that the effect of Re on the velocity and microrotation distribution is similar to that of $M$. The Reynolds number always tends to flatten the temperature profiles almost in the middle of the two disks, thus developing an equi-temperature region. On the other hand, it discourages thermal reversal near the lower disk, for the case $\mathrm{Ec} \neq 0$.

The effect of the micropolar structure of the fluid on the velocity, microrotation and temperature profiles is opposite to that of the magnetic field. Thus, the external magnetic field tends to balance the effect of micropolar parameters. The viscous dissipation tends to eliminate the symmetry of temperature profiles by raising them near the lower disks, thus causing the thermal reversal. Viscous dissipation plays a vital role like an internal heat generation source in the energy transfer, which depends on the temperature distributions and heat transfer rates. This heat source is caused by the shearing of fluid layers. The merit of the effect of the viscous dissipation depends on whether the disks walls are hot or cold. Finally, the Prandtl number increases the thermal reversal by increasing the temperature distribution across the disks in the presence of viscous dissipation (Fig. 6b).

Table 7. The effect of the Prandtl number on the heat transfer rate with $\operatorname{Re}=20, M=1$, $C_{1}=2, C_{2}=0.2, C_{3}=0.3$

\begin{tabular}{|c|c|c|c|c|}
\hline \multirow{2}{*}{$\operatorname{Pr}$} & \multicolumn{2}{|c|}{$\mathrm{Ec}=0.0$} & \multicolumn{2}{c|}{ Ec $=0.3$} \\
\cline { 2 - 5 } & $-\theta^{\prime}(-1)$ & $-\theta^{\prime}(1)$ & $-\theta^{\prime}(-1)$ & $-\theta^{\prime}(1)$ \\
\hline \hline 0.0 & 0.500000 & 0.500000 & 0.500000 & 0.500000 \\
\hline 0.2 & 0.719813 & 0.719813 & 0.494006 & 0.945619 \\
\hline 0.4 & 0.976928 & 0.976928 & 0.482008 & 1.471849 \\
\hline 0.6 & 1.254718 & 1.254718 & 0.426965 & 2.082470 \\
\hline 0.8 & 1.536267 & 1.536267 & 0.278411 & 2.794123 \\
\hline
\end{tabular}

On comparison of our results with those given by Khan et al. (2015) (where the classical Newtonian fluid has been taken into consideration between the two stretchable disks), we notice that the role of the external magnetic field and the disk stretching remains the same, even when the micropolar fluid is introduced in place of the classical Newtonian fluid. That is, both the factors increase the shear stresses at the disks. Micropolar fluids however show a remarkable reduction in the shear stress but introduce couple stresses at the disks due to the spinning of the fluid particles. 


\section{Conclusions}

In this paper, we numerically study how the governing parameters affect the flow and heat transfer characteristics of a steady laminar incompressible electrically conducting micropolar fluid between two stretchable infinite disks. The following conclusions can be drawn.

Micropolar fluids exhibit significant reduction in the shear stress at the disks compared to Newtonian ones, which may be beneficial for many industrial processes (e.g. in flow and thermal control of polymeric processing). The external magnetic field is responsible for a remarkable rise in both the shear and couple stresses while reduction the heat transfer rate at the two disks. We, therefore, conclude that the external magnetic field may serve as a controlling agent to neutralize the effects of the micropolar structure of the fluid. Thus, in experimental setups involving micropolar flows caused by moving disks, the possibility of interference of the external magnetic field should be eliminated in order to obtain accurate and reliable data.

\section{Acknowledgemment}

The authors are extremely grateful to the Higher Education Commission of Pakistan for the financial support to carry out this research. The authors are also very thankful to the learned reviewer for his remarks to improve the quality of the present work.

\section{References}

1. Ali K., Ashraf M., Jameel N., 2014, Numerical simulation of MHD micropolar fluid flow and heat transfer in a channel with shrinking walls, Canadian Journal of Physics, 92(2), 987-996

2. Altan T., Oh S., Gegel H.L., 1979, Metal Forming Fundamentals and Applications, American Society of Metals, Metals Park, $\mathrm{OH}$

3. Ashraf M., Batool K., 2013, MHD flow of a micropolar fluid over a stretching disk, Journal of Theoretical and Applied Mechanics, 51(1), 25-38

4. Ashraf M., Kamal M.A., Syed K.S., 2009a, Numerical simulation of a micropolar fluid between a porous disk and a non-porous disk, Journal of Applied Mathematics and Modelling, 33, 1933-1943

5. Ashraf M., Kamal M.A., Syed K.S., 2009b, Numerical study of asymmetric laminar flow of micropolar fluids in a porous channel, Computers and Fluids, 38, 1895-1902

6. Eringen A.C., 1964, Simple micropolar fluids, International Journal of Engineering Science, 2, $205-217$

7. Eringen A.C., 1965, Theory of Micropolar Continua, Proceedings of the Ninth Midwestern Conference

8. ERIngen A.C., 1966, Theory of micropolar fluids, Journal of Mathematics and Mechanics, 16, $1-18$

9. FAng T., Zhang J., 2008, Flow between two stretchable disks - An exact solution of the Navier-Stokes equations, International Communications in Heat and Mass Transfer, 35, 892-895

10. Fisher E.G., 1976, Extrusion of Plastics, Wiley New York

11. Hayat T., NAWAZ M., 2010, Effect of heat transfer on magnetohydrodynamic axisymmetric flow between two stretching sheets, Zeitschrift für Naturforschung, 65, 961-968

12. Hayat T., Nawaz M., Obaidat S., 2011, Axisymmetric magnetohydrodynamic flow of a micropolar fluid between unsteady stretching surfaces, Applied Mathematics and Mechanics, 32, 361-374

13. Hoyt J.W., Fabula A.G., 1964, The effect of additives on fluid friction, US Naval Ordinance Test Station Report 
14. Khan N., Sajid M., Mahmood T., 2015, Heat transfer analysis for magnetohydrodynamics axisymmetric flow between stretching disks in the presence of viscous dissipation and Joule heating, AIP Advances, 5, 057115

15. Munawar S., Mehmood A., Ali A., 2011, Effects of slip on flow between two stretchable disks using optimal homotopy analysis method, Canadian Journal of Applied Sciences, 1(2), 50-68

16. NeEtu S., 2014, MHD flow of the micropolar fluid between eccentrically rotating disks, International Scholarly Research Notices, Article ID-317075

17. Rashidi M.M., Kavyani N., Abelman S., 2014, Investigation of entropy generation in MHD and slip flow over a rotating porous disk with variable properties, International Journal of Heat and Mass Transfer, 70, 892-917

18. Robert A., Gorder V., Sweet E., Vajravelu K., 2010, Analytical solutions of a coupled nonlinear system arising in a flow between stretching disks, Applied Mathematics and Computation, 216, 1513-1523

19. Shercliff J.A., 1965, A Text Book of Magnetohydrodynamics, Pergamon Press Oxford

20. Tadmor Z., Klein I., 1970, Engineering Principles of Plasticating Extrusion, Polymer Science and engineering series, Van Norstrand Reinhold New York

21. Xinhui S., Liancun Z., Xinxin Z., Xinyi S., 2012, Homotopy analysis method for the asymmetric laminar flow and heat transfer of viscous fluid between contracting rotating disks, Applied Mathematical Modeling, 36, 1806-1820

Manuscript received January 30, 2015; accepted for print October 19, 2015 